\title{
Beyond Healthy Food Habits: Lessons Learned from a Community Kitchen and a Community Garden Developed by and for the Francophone and Francophile Minority of St. John's, Newfoundland
}

\author{
KamoRIAA
UNIVERSTY
${ }^{1}$ Memorial University of Newfoundland, Newfoundland ${ }^{2}$ Lakehead University, Ontario
}

\section{INTRODUCTION}

Literature abounds on the role of community kitchens and community gardens as strategies to foster food security and healthy living. ${ }^{1,2}$ However, very few studies have explored their unique contributions among minority communities in Canada. To our knowledge, no study to date have examined their impacts on Francophones and Francophiles living in a minority situation.

\section{OBJECTIFS}

Explore the benefits of a community kitchen and a community garden developed by and for the Francophone and Francophile minority living in St. John's, Newfoundland at an individual and a community level.

Identify key facilitators to their success

\section{METHODS}

Study design: An ethnographic approach including

$>$ Document consultation and overt participant observation done from September 2015 to July 2016.

In-depth semi-structured interviews $(n=15)$ done from April to July 2016. Interviewees included key informants and participants (men $n=2$, women $n=13$, Francophones $n=12$ and Francophiles $n=3$ ).

Data analysis: A thematic analysis was used.

\section{RESULTS}

Benefits: The community kitchen and garden play a key role in the development and vitality of the Francophone and Francophile community of St. John's. Francophones and Francophiles participate in these activities as they strongly resonate with their identity, values and interests. In addition to knowledge and skills gained, they enable participants to assert their identity; support the community; foster cultural and linguistic continuity; develop a strong sense of belonging; and provide cultural and linguistic safety and well-being.

Facilitators: Leadership, conviviality, the collective and hands on approach, connection to identity, exchanges between Francophones and Francophiles, and the use of French.

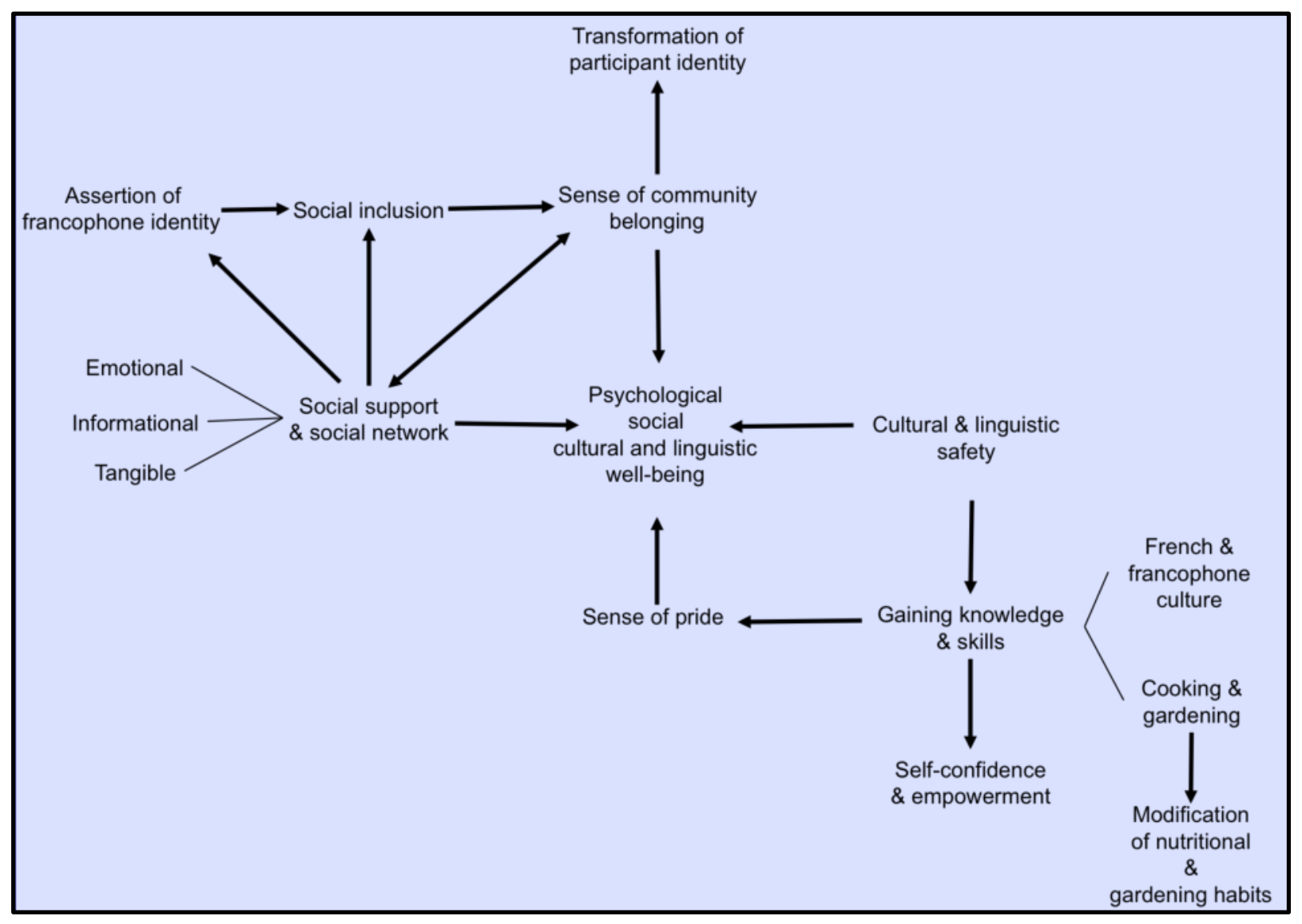

Figure 1. Results from the thematic analysis: Interactions between all benefits gained at the individual level.

1. Engler-Stringer, R., \& Berenbaum, S. (2005). Collective kitchens in Canada: A review of the literature. Canadian Journal of Dietetic Practice and Research, 66(4), 246-251. 2. Wakefield, S., Yeudall, F., Taron, C., Reynolds, J., \& Skinner, A. (2007). Growing urban health: Community gardening in South-East Toronto. Health Promotion International, 22(2), 92-101.

\section{DISCUSSION AND CONCLUSION}

Findings highlight the importance of considering both language and culture, as vital factors to participation and benefits gained. Results call for an expanded understanding of how community kitchens and gardens can contribute to individual and community well-being among minorities in Canada. This study points to the need for research that examines holistically how the well-being of individuals and communities can be fostered by health promotion, food movement and dietitian leaders.

\section{Recommendations to dietitians:}

Find ways to enable participants to assert their identity, and gain cultural and linguistic well-being.

Consider cultural and linguistic continuity and safety as crucial to foster cooking and gardening knowledge and skills.

\section{CHOSEN EXCERPTS FROM INTERVIEWS}

I love kitchen. I love eating ... As a member of the minority, in participating we help the community to grow. As a father, it also helps youths to feel a sense of belonging ... It is an extraordinary contribution (Fabien)

$>$ I interacted in French... It felt good! It brings my home here... Speaking French in an activity I love. When I came back [home] I was sooo excited! I was like: "Hey it was really fuuunnn!! I want to go back! » (Clara)

$>$ Gardening is a good opportunity for seniors to interact with children... which is important for cultural identity (Guylaine)

> I told myself : "Ah it's fun! ... Perhaps I will finally have the opportunity to be reminded [cooking] methods that I have grown with [as a child] » (Lise)

$>$ It [cooking] is already complicated in French, if on top of it it is in English (laugh) it would be too complicated... I like cooking, but not that much (Béatrix)

$>$ It's nice to be able to do a hands-on instead of watching it, right? (Rachel)

$>$ It is an ideal means to promote friendship, to promote, you know, it promotes communication, communication to be a friend, or, um, to learn French, or to learn how to cook (Ursula)

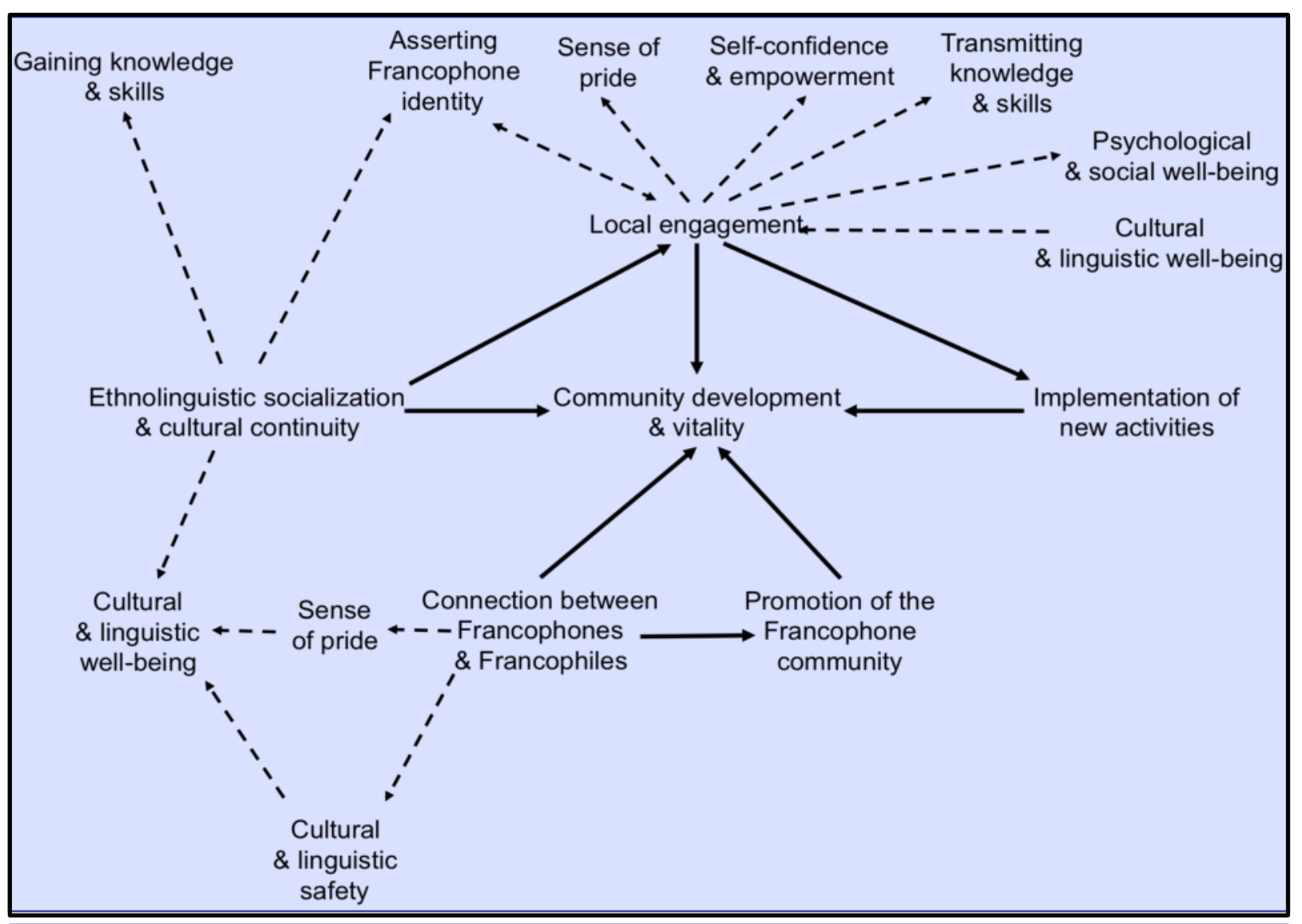

Figure 2. Results from the thematic analysis: Interactions among benefits gained at the community and individual level. Plain arrows: community level. Dashed arrows: between benefits at the individual and community level.

Copyright @ 2018 K.B. kb5557@mun.ca; R.S. rschiff@lakeheadu.ca; N.B. nbeausoleil@mun.ca 